\title{
Gute Praxis bricht formale Widerstände: Entwicklungschancen für das Europäische Sicherheits- und Verteidigungskolleg
}

\author{
Julia Lieb*
}

\section{Hohe Ziele - Verhaltene Mittel}

Die Idee eines Europäischen Sicherheits- und Verteidigungskollegs für die Europäische Union geht auf den Europäischen Rat von Köln 1999 zurück und wurde seitdem insbesondere von Frankreich und Deutschland vorangetrieben. ${ }^{1}$ Einen entscheidenden Impuls für die Umsetzung des Kollegs gab der sogenannte ,Pralinengipfel“ im April 2003 im belgischen Tervuren, auf dem sich Deutschland, Frankreich, Belgien und Luxemburg im Kontext der US-geführten Irakintervention für eine Stärkung der sicherheits- und verteidigungspolitischen Dimension der Europäische Union aussprachen. Der Gipfel der vier erklärten Gegner des Irakkrieges wurde teilweise als , antiamerikanische" Initiative und als Konkurrenz zur Fortentwicklung transatlantischer Strukturen aufgefasst. ${ }^{2}$ Beteuerungen seitens der Initiatoren, dass es sich allein um flankierende Maßnahmen in Übereinstimmung mit Zielen und Funktionen der NATO handle, konnten vehemente innereuropäische Kritiker wie beispielsweise Großbritannien zwar besänftigen, setzten dem Projekt allerdings klare Grenzen. So einigte sich der Ministerrat auf ein rein, virtuelles ' und damit sitzloses Kolleg mit schwachen Strukturen und ohne eigenes Budget.

Im November 2003 billigte der Ministerrat zunächst Eckdaten einer EU-Ausbildungspolitik für den Bereich der Europäischen Sicherheits- und Verteidigungspolitik (ESVP) und im September 2004 dann ein EU-Ausbildungskonzept. ${ }^{3}$ Ein erster ESVP-Pilotkurs fand 2004 noch vor der endgültigen Übertragung von Verantwortlichkeiten auf die europäische Ebene statt. ${ }^{4}$

Aufbauend auf der Arbeit verschiedener nationaler und europäischer Arbeitsgruppen konnte das Europäische Sicherheits- und Verteidigungskolleg (ESVK) im Juli 2005 durch eine Gemeinsame Aktion des Rates (GA) formell seine Arbeit aufnehmen. ${ }^{5}$ Erklärtes, ambitioniertes Ziel des Kollegs ist demnach die „Festigung einer Europäischen Sicherheitskultur" ${ }^{6}$ Den Ausbildungsteilnehmern soll dabei ein Verständnis für die diversen Aspekte der ESVP sowie deren Einbettung in den Gesamtkontext der Gemeinsamen Außen- und Sicherheitspolitik (GASP) vermittelt und der Sachverstand auf den verschiedenen Ebenen europä-

Julia Lieb, M.E.S., Villigst-Stipendiatin, Forschungsgruppe EU-Integration, Stiftung Wissenschaft und Politik, Berlin.

1 Vgl. Michel Monnier/Jean-Luc Cuny: Le Collège européen de sécurité et de défense: ,success story ‘?, in: Défense nationale et sécurité collective, Februar 2007, S. 21-30, hier S. 22-23.

2 Vgl. Washington Post: European Leaders Form Pact To Boost Defense Cooperation - Foes of Iraq War Seek to Operate Independendly of U.S., 30.04.2003.

3 Rat der EU: Entwurf einer EU-Ausbildungspolitik im Bereich der ESVP, Dok. 14520/03 vom 07.11.2003; Rat der EU: Entwurf eines EU Ausbildungskonzepts, Dok. 12035/04 vom 01.09.2004.

4 Monnier/Cuny: Le Collège européen de sécurité et de défense, 2007, S. 24.

5 Gemeinsame Aktion zur Einrichtung eines Europäischen Sicherheits- und Verteidigungskollegs, 2005/575/ GASP vom 18.07.2005, in: Amtsblatt der EU, Nr. L 194 vom 26.07.2005.

6 Gemeinsame Aktion, Artikel 3(a). 
ischer und nationaler Institutionen insgesamt erhöht werden. Zudem soll betroffenen Akteuren und Institutionen die Vernetzung und der Austausch erleichtert werden.

\section{Strukturen}

Das ESVK stellt ein Netzwerk ,nationaler Institute, Kollegs, Akademien und anderer Stellen, die sich innerhalb der EU mit Fragen der Sicherheits- und Verteidigungspolitik befassen" ${ }^{\text {"7 }}$ dar. Seitens der EU-Institutionen wird es durch das Institut für Sicherheitsstudien in Paris (EU ISS) unterstützt. Beteiligt sind unterschiedliche Einrichtungsformen aus einer Vielzahl von Mitgliedstaaten der Europäischen Union. (Siehe hierzu Tabelle 1.) Damit ist ein ungefähres Abbild der diversen in der Europäischen Union vorhandenen Sicherheitsund Verteidigungskulturen sowie eine gewisse inhaltliche Breite des ESVK gewährleistet.

Ein Lenkungsausschuss und ein akademischer Exekutivrat verantworten die Ausrichtung und Durchführung der Ausbildungsmaßnahmen. Ein ständiges ESVK-Sekretariat ist im Generalsekretariat des Rates der Europäischen Union angesiedelt. Die Verantwortung liegt hier allerdings vornehmlich bei einem einzelnen leitenden Beamten, der nur punktuell auf die Unterstützung des Politischen Stabs und der für die Außen- und Außenwirtschaftsbeziehungen zuständigen Generaldirektion E (Direktion 8 für Verteidigungsfragen) zurückgreifen kann. Eine insbesondere aus den Reihen des Kollegs geforderte stärkere Institutionalisierung des Sekretariats und eine angestrebte Personalaufstockung konnten in einer ersten Rekrutierungsrunde 2005 nicht realisiert werden. Letztere hätte durch die in der GA vorgesehene Entsendung von zwei nationalen Delegierten zur Verstärkung des ESVK-Sekretariats frühzeitig gewährleistet werden müssen. Die Mitgliedstaaten zeigten sich hier allerdings zunächst nur wenig bereit, geeignete nationale Experten zu entsenden und zu finanzieren. Im August 2007 wurde nun aber eine nach Ansicht Beteiligter vielversprechende neue Auswahlrunde mit qualifizierten Kandidaten eingeleitet. ${ }^{8}$

An den Ausbildungsmaßnahmen sowie an deren Durchführung beteiligen sich das Ratssekretariat, die Europäische Kommission und das Europäische Parlament. Deren Referenten und Räumlichkeiten tragen zur erfolgreichen Umsetzung der Ausbildungsprogramme - insbesondere der Maßnahmen in Brüssel - bei. Die Kontakte des ständigen Sekretariats zur EU-Kommission sind regelmäßig und nach Angaben Beteiligter konstruktiv. ${ }^{9}$ Kommissionsvertreter der Generaldirektion RELEX (Außenbeziehungen) sind hier in ein kleines informelles ,ESVP-Ausbildungs-Team‘ im Ratssekretariat eingebunden. Das ESVK kann damit auf funktionierende, aber eben sehr schwach institutionalisierte Strukturen aufbauen. ${ }^{10}$

Die Finanzierung des ESVK wird durch die Mitgliedstaaten und die beteiligten Einrichtungen auf nationaler und europäischer Ebene sowie durch die Teilnehmer der Ausbildungsprogramme gewährleistet. Auf mitgliedstaatlicher Seite ist die Bereitschaft, die einzelnen Ausbildungsmaßnahmen mit finanziellen Mitteln auszustatten sehr unterschiedlich ausgeprägt. Eine Finanzierung des Kollegs aus dem EU-Haushalt wurde bislang nicht in Erwägung gezogen. Seine Möglichkeiten bleiben damit klar begrenzt. ${ }^{11}$

7 Gemeinsame Aktion, Artikel 1(2).

8 Informationen aus dem Generalsekretariat des Rates der EU. Vgl. zudem Jahresbericht des ESVK 2006, SC/2006/ 07 REV 1 vom 12.03.2006, S. 3 und Jahresbericht des ESVK 2007, SC/2007/010 REV 1 vom 28.03.2007, S. 6.

9 Vgl. Jahresbericht 2006, S. 4; Jahresbericht 2007, S. 2.

10 Informationen aus dem Generalsekretariat des Rates der EU.

11 Gemeinsame Aktion, Artikel 11. Vgl. auch Monnier/Cuny: Le Collège européen de sécurité et de défense, 2007, S. 26. 
Tabelle 1: Am ESVK bisher beteiligte mitgliedstaatliche Institute

\begin{tabular}{|c|c|c|c|}
\hline Land & Einrichtung & Homepage & Sitz \\
\hline $\begin{array}{l}\text { Baltische } \\
\text { Staaten }\end{array}$ & $\begin{array}{l}\text { Baltic Defence College / Baltisches } \\
\text { Verteidigungskolleg }\end{array}$ & http://www.bdcol.ee & Tartu \\
\hline Belgien & $\begin{array}{l}\text { Institut Royal Supérieur de Défense } \\
\text { (IRSD/KHO) / Königliches Verteidi- } \\
\text { gungsinstitut }\end{array}$ & http://www.mil.be/rdc & Brüssel \\
\hline Deutschland & $\begin{array}{l}\text { Bundesakademie für Sicherheitspoli- } \\
\text { tik (BAKS) }\end{array}$ & http://www.baks.bundeswehr.de & Berlin \\
\hline Finnland & $\begin{array}{l}\text { Tervetuloa Maanpuolustuskorkeakou- } \\
\text { luun / Nationale Verteidigungsuniver- } \\
\text { sität }\end{array}$ & http://www.mpkk.fi & Helsinki \\
\hline Frankreich & $\begin{array}{l}\text { Institut des Hautes Études de Défense } \\
\text { Nationale (IHEDN) / Nationales Insti- } \\
\text { tut für Verteidigungsstudien }\end{array}$ & http://www.ihedn.fr & Paris \\
\hline $\begin{array}{l}\text { Griechen- } \\
\text { land }\end{array}$ & $\begin{array}{l}\text { Diplomatische Akademie und Natio- } \\
\text { nales Verteidigungskolleg }\end{array}$ & www.mfa.gr / www.setha.mil.gr & Athen \\
\hline $\begin{array}{l}\text { Groß- } \\
\text { britannien }\end{array}$ & $\begin{array}{l}\text { Defence Academy of the United King- } \\
\text { dom / Britische Verteidigungsakade- } \\
\text { mie }\end{array}$ & http://www.defac.ac.uk & Shrivenham \\
\hline Italien & $\begin{array}{l}\text { Istituto Superiore di Stato Maggiore } \\
\text { Interforze (ISSMI) / Nationales Ver- } \\
\text { teidigungsinstitut }\end{array}$ & $\begin{array}{l}\text { http://www.difesa.it/SMD/CASD/Isti- } \\
\text { tuti+militari/ISSMI }\end{array}$ & Rom \\
\hline Niederlande & $\begin{array}{l}\text { Institut Defensie Leergangen (IDL) / } \\
\text { Niederländische Verteidigungsakade- } \\
\text { mie }\end{array}$ & $\begin{array}{l}\text { http://www.nederlandsedefensieaca- } \\
\text { demie.nl }\end{array}$ & Den Haag \\
\hline Österreich & Landesverteidigungsakademie & $\begin{array}{l}\text { http://www.bundesheer.at/organisa- } \\
\text { tion/beitraege/lvak }\end{array}$ & Wien \\
\hline Polen & $\begin{array}{l}\text { Akademia Obrony Narodowej / Natio- } \\
\text { nale Verteidigungsakademie }\end{array}$ & www.aon.edu.pl & Warschau \\
\hline Portugal & $\begin{array}{l}\text { Instituto da Defesa Nacional / Natio- } \\
\text { nales Verteidigungsinstitut }\end{array}$ & http://www.idn.gov.pt & Lissabon \\
\hline Rumänien & $\begin{array}{l}\text { Colegiul National de Aparare / Natio- } \\
\text { nales Verteidigungskolleg }\end{array}$ & $\begin{array}{l}\text { http://www.unap.ro/ro/unitati/cnap/ } \\
\text { cnap.html }\end{array}$ & Bukarest \\
\hline Schweden & $\begin{array}{l}\text { Försvarshögskolan / Nationales Ver- } \\
\text { teidigungskolleg }\end{array}$ & http://www.fhs.se/sv & Stockholm \\
\hline Slowenien & $\begin{array}{l}\text { Ministrstvu za obrambo / Nationales } \\
\text { Verteidigungsministerium (Institut) }\end{array}$ & http://www.mors.si & Ljubljana \\
\hline Spanien & $\begin{array}{l}\text { Centro Superior de Estudios de la De- } \\
\text { fensa Nacional (CESEDEN) / Zen- } \\
\text { trum für Nationale Sicherheitsstudien }\end{array}$ & http://www.ceseden.es & Madrid \\
\hline Tschechien & $\begin{array}{l}\text { Vítejte na Univerzitì obrany / Natio- } \\
\text { nale Verteidigungsuniversität }\end{array}$ & http://www.unob.cz & Brno \\
\hline Ungarn & $\begin{array}{l}\text { Zrínyi Miklós Nemzetvédelmi Egyetem / } \\
\text { Nationale Verteidigungsakademie }\end{array}$ & http://www.zmne.hu & Budapest \\
\hline
\end{tabular}

Quelle: Generalsekretariat des Rates der Europäischen Union 


\section{Ausbildung}

Hauptaufgabe des ESVK ist es, Ausbildungsmaßnahmen der Europäischen Union im strategischen Bereich der ESVP in einem umfassenden Ansatz zu organisieren und durchzuführen. ${ }^{12}$ Hierfür werden insbesondere zwei Ausbildungsformen angeboten: ESVP-Lehrgänge auf hohem Niveau (sogenannte High-Level Courses, HLC) und Orientierungsseminare. ${ }^{13}$

Die Orientierungskurse dienen der Erlangung grundlegender Kenntnisse über die ESVP. Vier bis fünf jeweils wöchentliche Seminare für circa 60 Teilnehmer werden jährlich vorzugsweise in Brüssel durchgeführt, da hier die Kosten gering gehalten und die Nähe zu relevanten Akteuren für die Ausbildungsmaßnahmen genutzt werden können. Die Seminare richten sich an ein breites Teilnehmerfeld von Angehörigen der Mitgliedstaaten und Beitrittskandidaten der Europäischen Union (in Deutschland auf der A13/A14-Ebene) aber seit Kurzem auch Vertretern von Drittstaaten und internationalen Organisationen. Diese Öffnung des Programms wurde unter österreichischer EU-Ratspräsidentschaft erwirkt und allgemein als positiv bewertet. ${ }^{14}$

Der Teilnehmerkreis der HLC, die das Flaggschiff des ESVK darstellen, ist enger gefasst. Hier soll mitgliedstaatliches Führungspersonal aus dem zivilen und militärischen Bereich, das mit strategischen Aspekten der ESVP in Berührung kommt und Karriereambitionen in diesem Feld hat, angesprochen werden. Auch Angehörigen von EU-Institutionen steht die Teilnahme offen. Die Lehrgänge werden in fünf einwöchigen Modulen über einen Zeitraum von sieben Monaten und für wiederum circa 60 Teilnehmer angeboten. Die beteiligten Einrichtungen halten die Kurse in der Regel jeweils an ihrem Sitz ab, teilweise in Brüssel und auch in Kooperation mit weiteren Institutionen des Netzwerks. Die fünf Module des HLC bauen inhaltlich aufeinander auf und gliedern sich in die Themenfelder Grundlagen der ESVP, Krisenmanagementfähigkeiten, Krisenmanagementoperationen, ESVP im regionalen Kontext und Zukunft der ESVP. (Zur Ausrichtung der HLC im Zeitraum 2006-2008 siehe Tabelle 2.)

Die Inhalte und der Ablauf der beiden Ausbildungsmaßnahmen werden von den Beteiligten insgesamt als positiv gewertet. Dennoch unterstreichen die regelmäßigen Evaluierungen der Programme auch, dass die Standardcurricula der Seminare und Module weiter verfeinert und den Bedürfnissen der jeweiligen Teilnehmer angepasst werden müssten. Ebenso stellt sich die Herausforderung einer zielgerichteten und ausgewogenen Zusammenführung ziviler und militärischer Aspekte und Teilnehmer im Kolleg. In den Reihen des ESVK selbst wird das gegenwärtige Übergewicht militärischer Elemente in der Ausbildung durchaus eingestanden. ${ }^{15}$

Der begrenzte Wirkungsbereich des ESVK ist sicher auf die ressourcenbedingten geringen Teilnehmerzahlen zurückzuführen. ${ }^{16}$ Innerhalb des Kollegs werden nun neue Ausbildungsinhalte und -formen diskutiert und teilweise umgesetzt. Nach einem Pilotkurs für führendes Personal der Presse- und Öffentlichkeitsarbeit nationaler Regierungseinrichtungen

12 Vgl. Hans-Bernhard Weißerth: Das Europäische Sicherheits- und Verteidigungskolleg: wesentliches Instrument zur Weiterentwicklung der europäischen Sicherheits- und Verteidigungspolitik, in: Sicherheit und Frieden 1/2007, S. 11-14, hier S. 12.

$13 \mathrm{Zu}$ den folgenden Erläuterungen siehe insbesondere Weißerth: Das Europäische Sicherheits- und Verteidigungskolleg, 2007, S. 13-14 sowie den Entwurf über die Modalitäten der Arbeitsweise des ESVK, Rat der EU, Dok. 9184/2/05 REV 2, S. 7 ff.

14 Vgl. Jahresbericht 2006, S. 5.

15 Vgl. Jahresbericht 2006, S. 4-5; Jahresbericht 2007, S. $1 \mathrm{ff}$.

16 Vgl. Weißerth: Das Europäische Sicherheits- und Verteidigungskolleg, 2007, S. 14. 
im November 2006 wurde diese neue Ausbildungsform als fester Bestandteil in die kommenden Jahresprogramme des ESVK übernommen. ${ }^{17}$

Nachgedacht wird über Intensivkurse für hochrangige Entscheidungsträger sowie für die Sonderbeauftragten der Europäischen Union, betroffene Missionsleiter und militärische Kommandierende. Im Gespräch sind zudem eine Ausweitung der Ausbildungsmaßnahmen auf Rechtsberater und politische Berater im Bereich der Sicherheits- und Verteidigungspolitik als auch eine Fokussierung auf spezifische Regionen oder horizontale Themen.

Tabelle 2: Ausrichtung der ESVP High-Level Kurse des ESVK im Zeitraum 2006-2008

\begin{tabular}{|c|c|c|c|}
\hline Modul & Ort & Ausrichtend & $\begin{array}{c}\text { Unterstützend } \\
\text { (finanziell/ } \\
\text { logistisch) }\end{array}$ \\
\hline \multicolumn{4}{|l|}{ 1. Einführungsseminar } \\
\hline $2006-2007$ & Brüssel & $\begin{array}{l}\text { IRSD/KHID, Ratssekretariat und } \\
\text { Kommission }\end{array}$ & $\begin{array}{l}\text { Niederlande } \\
\text { Luxemburg }\end{array}$ \\
\hline $2007-2008$ & Brüssel & $\begin{array}{l}\text { IRSD/KHID, Ratssekretariat und } \\
\text { Kommission }\end{array}$ & $\begin{array}{l}\text { Niederlande } \\
\text { Luxemburg }\end{array}$ \\
\hline \multicolumn{4}{|c|}{ 2. Krisenmanagementfähigkeiten } \\
\hline 2006-2007 & Paris & IHEDN & \\
\hline $2007-2008$ & Lissabon & Portugiesisches Verteidigungsinstitut & BAKS \\
\hline \multicolumn{4}{|c|}{ 3. Krisenmanagementeinsätze } \\
\hline 2006-2007 & Athen & $\begin{array}{l}\text { Griechische diplomatische Akademie } \\
\text { und nationales Verteidigungskolleg }\end{array}$ & $\begin{array}{l}\text { Italien } \\
\text { Österreich }\end{array}$ \\
\hline $2007-2008$ & Prag & Tschechische Verteidigungsuniversität & Großbritannien \\
\hline \multicolumn{4}{|c|}{ 4. ESVP im regionalen Kontext } \\
\hline $2006-2007$ & Madrid & CESEDEN & $\begin{array}{l}\text { Polnische } \\
\text { diplomatische } \\
\text { Akademie }\end{array}$ \\
\hline $2007-2008$ & Tartu & Baltisches Verteidigungskolleg & $\begin{array}{l}\text { Österreichi- } \\
\text { sche Verteidi- } \\
\text { gungsakademie }\end{array}$ \\
\hline \multicolumn{4}{|l|}{ 5. Zukunft der ESVP } \\
\hline 2006-2007 & Berlin & BAKS & \\
\hline $2007-2008$ & Ljubljana & $\begin{array}{l}\text { Slowenisches nationales } \\
\text { Verteidigungsinstitut }\end{array}$ & \\
\hline
\end{tabular}

Quelle: EU Trainingsmaßnahmen im Bereich der ESVP 2006-2009, von EU-Organen und Mitgliedstaaten ausgerichtete Kurse, abrufbar unter: www.consilium.europa.eu (letzter Zugriff: 08.08.2007).

\section{Wille und Weg zur Revision der Gemeinsamen Aktion}

Die schwache Institutionalisierung und die begrenzten Möglichkeiten des ESVK stehen im Gegensatz zur steigenden Nachfrage nach dessen Ausbildungsprogrammen. Insgesamt

17 Informationen aus dem Generalsekretariat des Rates der EU. Vgl. zudem Jahresbericht 2007, S. 3. 
übersteigen die Teilnehmerbewerbungen - die auch immer zahlreicher aus Großbritannien eintreffen - regelmäßig die vorhandenen Kapazitäten. Die von Beteiligten als ,gut bis hervorragend" bewertete Ausbildung und der Wirkungsbereich des ESVK hat damit wohl einige der ursprünglichen Widerstände beseitigt. ${ }^{18}$

Unter portugiesischer EU-Ratspräsidentschaft wird nun, wie in Artikel 13 der GA vorgesehen, ein Bericht über die „Tätigkeiten und Perspektiven des ESVK“ erarbeitet und an den Rat übermittelt. Dieser Bericht soll dann als Grundlage für eine Revision der GA dienen, die bis zu deren Auslaufen im Juli 2008 unter slowenischer Präsidentschaft beschlossen und unter französischer Präsidentschaft im zweiten Halbjahr 2008 umgesetzt werden müsste. ${ }^{19}$

Die Überarbeitung der GA könnte zur Einrichtung stärker belastbarer, permanenter Strukturen und hiermit einhergehend zur Lösung der Personal- und Haushaltsprobleme genutzt werden, sowie die Frage der Rechtspersönlichkeit klären und weitere Defizite des ESVK beheben. Ebenso besteht aber die Möglichkeit, den Status quo fortzuschreiben oder die Aktivitäten des ESVK zurückzufahren. Eine Zurückstufung oder gar Abschaffung des Kollegs steht aufgrund seines bisherigen Erfolgs zwar nicht zur Debatte. Allerdings sträuben sich insbesondere Großbritannien und die Tschechische Republik gegen einen Ausbau permanenter Strukturen. ${ }^{20}$

Die Schlüsselfrage wird damit sicherlich die Haltung Großbritanniens in den Verhandlungen darstellen. Die britische Seite wäre in diesem Zusammenhang daran zu erinnern, dass sie einer der aktivsten Nutzer des ESVK ist. ${ }^{21}$ Ein sicher nicht uninteressanter weiterer Hinweis für die erweiterungsfreudigen Briten wäre die mögliche Einbindung von Kandidatenländern und Drittländern in die Ausbildungsmaßnahmen des Kollegs, um die Teilnehmer aus diesen Staaten rasch an den politischen Besitzstand der Europäischen Union heranzuführen. Ebenso könnte die verstärkte Kooperation des ESVK mit anderen sicherheits- und verteidigungspolitischen Instituten wie dem NATO-Verteidigungskolleg helfen, britische Vorbehalte zu überwinden.

Wahrscheinlich ist ein Einlenken Londons aber nur, wenn es gelingt, den konkreten Mehrwert einer stärkeren Strukturierung des ESVK stichhaltig zu begründen. Zudem müsste dem Eindruck entgegengewirkt werden, dass eine Institutionalisierung des Kollegs die nationale Autonomie der Aus- und Fortbildungspolitik Großbritanniens in der Sicherheitsund Verteidigungspolitik in Frage stellt. Die Verhandlungen zum ESVK werden parallel zur Regierungskonferenz über den Reformvertrag geführt. Großbritannien hat im Mandat hierzu zwei rechtlich unverbindliche Erklärungen durchgesetzt, die die Wahrung der nationalen Eigenständigkeit in Fragen der Außen- und Sicherheitspolitik betonen. ${ }^{22}$ Im Hinblick auf die Ratifikation des neuen Reformvertrages wäre daher auch bei den ESVK-Beratungen darauf zu achten, dass diese britischen Sonderanliegen respektiert werden.

Stellt sich bei den Gesprächen zum ESVK heraus, dass unüberbrückbare Differenzen zwischen den Verhandlungspartnern bestehen, so erscheint die Beibehaltung des Status quo als einzig gangbarer Ausweg, um sich langfristig - in Erwartung der mit dem Reformvertrag rechtlich verbindlich sanktionierten Reformen der GASP und der ESVP - mit einer grundlegenden Neuauflage des Kollegs auseinanderzusetzen. Wünschenswert wäre es in diesem

18 Vgl. Jahresbericht 2006, S. 5; Jahresbericht 2007, S. 1-2, 4 und $11 \mathrm{ff}$.

19 Vgl. Monnier/Cuny: Le Collège européen de sécurité et de défense, 2007, S. 28ff. (Der Zeitplan der beiden Autoren wurde für diesen Artikel aktualisiert).

20 Informationen am ESVK Beteiligter.

21 Vgl. Jahresbericht 2007, S. $11 \mathrm{ff}$.

22 Siehe Mandat für die Regierungskonferenz 2007, Rat der EU, Dok. 11218/07 vom 26.06.2007. 
Fall, zumindest konsensfähige institutionelle Verbesserungen für die laufende Arbeit des Kollegs zu vereinbaren (Personalaufstockung des Sekretariats).

\section{Entwicklungschancen}

Insgesamt bieten sich für eine Beschleunigung der Reform des ESVK zahlreiche Anknüpfungspunkte an. Diese sollten von den das ESVK befürwortenden Akteuren in die Verhandlungen eingebracht werden.

\section{Institutionalisierung vorantreiben}

Die Schaffung eines personell und institutionell vollwertigen ständigen Sekretariats ist eine notwendige Bedingung für die Weiterentwicklung und Identität des ESVK. ${ }^{23}$ Nur eine zentrale Koordinierung seiner Aktivitäten garantiert eine kohärente Ausrichtung des Kollegs und seine Sichtbarkeit im EU-Gefüge. Administrative Zentralisierung und operative Vernetzung nationaler Institute könnten sich hierbei ergänzen. Die mitgliedstaatlichen Teilnehmerinstitute würden bei dieser autonomieschonenden Reform weiterhin die Hauptverantwortung für die Ausbildungsmaßnahmen tragen und durch eine zentrale Koordinierungs-, Planungs- und Bewertungsstelle entlastet werden. Die nationalen Ausbildungsinstitute könnten von dieser Konstellation zugleich einen Zugewinn an Expertise sowie eine stärkere Einbindung in aktuelle ESVP-Entwicklungen erwarten.

Um Rolle und Auftrag des ESVK zu stärken, sollte der Personalpolitik aber auch der Frage der institutionellen Verankerung und der Rechtspersönlichkeit des ESVK größere Bedeutung beigemessen werden. Klärungsbedürftig wäre in diesem Zusammenhang ebenso die Repräsentation des Kollegs nach außen durch einen eindeutig dafür bestimmten Vertreter. Verbesserungswürdig sind zudem die Entscheidungsverfahren im Lenkungsausschuss und im akademischen Exekutivrat. Diese gestalten sich teilweise als ineffektiv und zu zeitinten$\operatorname{siv}^{24}$

\section{Materielle und ideelle Unterstützung}

Eine Ausweitung der Ausbildungsmaßnahmen und Aktivitäten des Kollegs ist sicherlich nur mit einer Aufstockung finanzieller und personeller Ressourcen zu gewährleisten. Die Mitgliedstaaten als Hauptfinanzierer des Kollegs zeigten sich hier in der Vergangenheit häufig sehr zurückhaltend. Wenn eine Finanzierung des Kollegs künftig nicht aus dem EUHaushalt gewährleistet werden kann, müsste der Verweis auf die bisherigen Leistungen des ESVK den notwendigen politischen Willen erzeugen, um in den Mitgliedstaaten entsprechend Mittel zu generieren.

Die Wirkung politischer Symbole sollte für einen Erfolg des ESVK nicht unterschätzt werden, denn ein Teil der bisherigen Publizität des Kollegs im Bereich der ESVP geht auch auf dessen Unterstützung von höchster Ebene zurück. So spricht der Hohe Vertreter und Generalsekretär des Rates Javier Solana jeweils die Einladungen für die HLC aus und überreicht den Teilnehmern wenn möglich im Rahmen einer feierlichen Veranstaltung ihre Abschlusszertifikate. ${ }^{25}$

23 Vgl. Jahresbericht 2006, S. 6; Jahresbericht 2007, S. 6-7.

24 Vgl. Jahresbericht 2006, S. 6; Jahresbericht 2007, S. 5ff.

25 „Javier Solana, Hoher Repräsentant der EU für die GASP gratuliert dem Europäischen Sicherheits- und Verteidigungskolleg zu seinem ersten Geburtstag“, Rat der Europäischen Union, Pressemitteilung S213/06. 


\section{Balance der Ausbildung garantieren}

Die beschriebene modulare Ausbildung hat sich als erfolgreich erwiesen. Bei den HLC kommen die Zeitabstände von ein bis zwei Monaten zwischen den einzelnen Modulen und deren Umfang den Teilnehmern in ihren sonstigen Verpflichtungen entgegen und vermitteln ausreichend Lerninhalte. ${ }^{26}$

Neben der erwähnten kollegsinternen Diskussion über die Ausweitung des Ausbildungsangebots beziehen sich Vorschläge aus den Reihen des ESVK aber auf eine verbesserte inhaltliche und organisatorische Ausrichtung der Lehrpläne. Sie betreffen eine langfristigere Planung der Ausbildungsmaßnahmen, die Formulierung vergleichbarer Ausbildungsstandards in den Lehrplänen und deren Veröffentlichung, den Aufbau eines Pools ständiger und erfahrener Seminarleiter und die ausgeglichene Auswahl sowohl der inhaltlichen Ausbildungselemente als auch der Teilnehmer aus dem zivilen und dem militärischen Bereich. ${ }^{27}$

$\mathrm{Zu}$ begrüßen sind die Bemühungen innerhalb des Kollegs, derartige Punkte unter der Zielsetzung einer Formulierung eines Gesamtkonzepts zu diskutieren. ${ }^{28}$ Für eine tatsächliche Verstärkung und Zusammenführung von Ausbildungsmaßnahmen im sicherheits- und verteidigungspolitischen Bereich, müssten die Mitgliedstaaten allerdings eine angemessene Finanzierung gewährleisten.

\section{Möglichkeiten des Internets nutzen}

Entgegen der ursprünglichen Absichten der GA spielt das Internet als Ausbildungsplattform aufgrund der begrenzten Mittel derzeit in den Ausbildungsaktivitäten des ESVK eine marginale Rolle. ${ }^{29}$ Mit dem Internet könnten allerdings in zweifacher Weise Verbesserungen herbeigeführt werden: Zum einen bietet webbasiertes Lernen eine kostengünstige Möglichkeit zur Ausweitung von Ausbildungsprogrammen. Zum anderen erlaubt es eine stärkere Außenwirkung des Kollegs und die gleichzeitige dauerhafte Vernetzung beteiligter Akteure und Einrichtungen. Die bereits praktizierte stärkere Zusammenarbeit mit in dieser Hinsicht erfahrenen Partnern wie der Königlichen Verteidigungsakademie Belgiens ist ein erster wichtiger Schritt zum Ausbau der Kapazitäten in diesem Bereich. ${ }^{30}$

\section{Netzwerkcharakter ausbauen}

Die am ESVK partizipierenden Einrichtungen stehen durch die Zusammenarbeit im Akademischen Exekutivrat, die Abstimmung von Ausbildungsprogrammen und der gelegentlich praktizierten gemeinsamen Durchführung von Ausbildungsmodulen der HLC in regelmäßigem Austausch. Allerdings sollte der unzureichenden Kontinuität in der Ausrichtung der Ausbildungsmaßnahmen des Kollegs durch verbindlichere und langfristigere Partizipationszusagen sowie der dauerhaften Einplanung nationaler Ressourcen entgegen gewirkt werden. Auch in der Intensivierung etablierter Kontakte und der Vergrößerung und Diversifizierung des ESVP-Netzwerks über die Grenzen der Europäischen Union hinaus besteht weiteres

26 Vgl. Jahresbericht 2006, S. 3; Jahresbericht 2007, S. 2.

27 Vgl. Jahresbericht 2006, S. 4-5; Jahresbericht 2007, S. 1-2.

28 Informationen aus dem Generalsekretariat des Rates der EU. Vgl. auch Jahresbericht 2006, S. 5; Jahresbericht 2007, S. 3.

29 Bislang können auf der Seite des Rates der EU, abrufbar unter: www.consilium.europa.eu (letzter Zugriff: 11.09.2007), allein die Ausbildungsprogramme im gesamten Bereich der ESVP seit 2006 eingesehen werden. Zudem steht dem ESVK nicht, wie beispielsweise dem European Diplomatic Programme (EDP), ein Intranet zur Vermittlung von Lerninhalten oder der Pflege von Kontakten zur Verfügung. (Das EDP ist ein gemeinsames Projekt von Mitgliedstaaten und EU-Organen und im Bereich der GASP angesiedelt).

30 Vgl. Jahresbericht 2007, S. 3. 
Entwicklungspotenzial. In diesem Zusammenhang wäre ebenso über konkrete Anknüpfungspunkte an andere ESVP-Ausbildungsmaßnahmen (zum Beispiel im militärischen und zivil-militärischen oder im polizeilichen Bereich) nachzudenken. Die ohnehin regelmäßig stattfindenden Treffen der beteiligten Einrichtungen an den vielfältigen Ausbildungsmaßnahmen unter dem Dach der ESVP sind hier bereits richtungsweisend. ${ }^{31}$ Nicht zu unterschätzen ist zudem der Mehrwert des Aufbaus eines Alumni-Netzwerks zur Pflege von Kontakten ehemaliger Ausbildungsteilnehmer.

\section{Schlussfolgerungen und Ausblick}

Das ESVK ist zu einem ,entscheidenden Akteur für die Ausbildung im Bereich der ESVP“32 geworden. Es trägt zur Herausbildung und Stärkung einer europäischen Sicherheitskultur bei, die für eine handlungsfähige ESVP sowie die internationale Zusammenarbeit unabdingbar geworden ist. ${ }^{33}$ Eine ambitionierte Weiterentwicklung des Kollegs könnte auch für andere Ausbildungsmaßnahmen im außen- und sicherheitspolitischen Bereich der Europäischen Union Modellcharakter erlangen und die positive Wirkung praktischer Zusammenarbeit unterstreichen. Gleichwohl müssten sich die Mitgliedstaaten der Europäischen Union über die langfristigen Zielsetzungen einigen, die mit dem ESVK verfolgt werden sollen. Damit wären auch die grundlegenden Fragen des Verhältnisses des Kollegs zu bestehenden nationalen Ausbildungsmaßnahmen sowie der künftig notwendigen und wünschenswerten Inhalte und Prioritäten europäischer Ausbildung insgesamt aufgeworfen.

In den Verhandlungsrunden unter portugiesischer und slowenischer EU-Ratspräsidentschaft wird es bis Mitte 2008 darum gehen, die genannten institutionellen und ausbildungstechnischen Entwicklungschancen soweit wie möglich zu nutzen und offensichtliche Defizite zu beseitigen. Die Umsetzung einzelner Maßnahmen - wie beispielsweise eine angemessene und gesicherte Finanzierung des Kollegs über den EU-Haushalt - könnten kollegsinterne Spannungen und mitgliedstaatliche Vorbehalte abbauen. Allerdings sollte dabei die Formulierung eines belastbaren und zukunftsfähigen Gesamtkonzepts für das ESVK nicht aus den Augen verloren werden.

Den zu erwartenden britischen Widerständen sollten die Befürworter des Kollegs im Verbund begegnen. Das deutsch-französische Tandem stellt dabei nur eine Möglichkeit geeinten Auftretens dar. Auch die gegenwärtige Triopräsidentschaft von Deutschland, Portugal und Slowenien könnte hier Führungswillen zeigen und damit gleichzeitig den Interessen der kleineren Mitgliedstaaten an einer Konsolidierung der ESVP-Strukturen Rückendeckung geben. Das Europäische Parlament wäre nicht zuletzt als Teil der Haushaltsbehörde und Verfechter eines Ausbaus des ESVK frühzeitig in die Reformüberlegungen einzubeziehen.

Weiterer Reformdruck könnte schließlich mit der Erhöhung des Bekanntheitsgrades des Kollegs aufgebaut werden. Dies wäre durch eine intensivere öffentliche Unterstützung von hoher politischer Ebene zu erreichen, die gerade in den einzelnen Mitgliedstaaten noch stark ausbaufähig ist.

31 Vgl. Jahresbericht 2006, S. 7.

32 Weißerth: Europäisches Sicherheits- und Verteidigungskolleg, 2007, S. 12; Vgl. zudem Jahresbericht 2007, S. 7.

33 Vgl. Hans-Georg Erhardt: The EU as a civil-military crisis manager: Coping with internal security governance, in: International Journal 2/2006, S. 433-450. 